\title{
Effect of DNA methylation on gene transcription is associated with the distribution of methylation sites across the genome in osteoarthritis
}

\author{
PENG YI $^{1^{*}}, \mathrm{XIONGFENG} \mathrm{XU}^{2 *}$, JIAWEI YAO ${ }^{1}$ and $\mathrm{BO} \mathrm{QIU}^{1}$ \\ Departments of ${ }^{1}$ Orthopedics and ${ }^{2}$ Emergency, Renmin Hospital of Wuhan University, Wuhan, Hubei 430060, P.R. China
}

Received October 23, 2020; Accepted March 26, 2021

DOI: $10.3892 /$ etm.2021.10151

\begin{abstract}
Genetics and epigenetics are important subjects in the field of osteoarthritis (OA) research. DNA methylation may affect gene transcription, but the specific mechanisms have remained to be fully elucidated. In the present study, the ChAMP methylation analysis package was used to identify differentially methylated genes (DMGs) from the dataset GSE63695 from the Gene Expression Omnibus (GEO) database. The distribution of differentially methylated sites (DMS) and the total array sites across the genome were analyzed by enrichment analysis. Subsequently, two mRNA expression profiling datasets, GSE114007 and GSE113825, were obtained from the GEO database and common differentially expressed genes (DEGs) were identified using the Limma package. Key genes were screened by analyzing the distribution of DMS across the genome consisting of DEGs and DMGs. A total of 1,662 and 1,986 DEGs were identified between OA and normal human cartilage from the GSE113825 and GSE114007 dataset, respectively. A further screening revealed 292 genes with common differences between the two datasets. A total of 574 DMS containing 394 DMGs were observed between OA and normal cartilage. Integrative analysis revealed a corresponding subset of 15 genes. Of these, 6 genes were verified by reverse transcription-quantitative PCR, confirming that the mRNA expression of 5 genes (MAP1B, FNDC1, ANLN, SCNN1A and STC2) in OA cartilage was consistent with the mRNA expression from the analysis of the datasets. Upon treatment with the DNA methylation inhibitor 5-aza-2'-deoxycytidine, the mRNA levels of FNDC1 and SCNN1A were decreased, and no significant alteration in the mRNA levels of MAP1B, ANLN, KCNN4 and STC2 was observed. The incidence of differential methylation
\end{abstract}

Correspondence to: Professor Bo Qiu, Department of Orthopedics, Renmin Hospital of Wuhan University, 238 Jiefang Road, Wuhan, Hubei 430060, P.R. China

E-mail: qbtg163@163.com

*Contributed equally

Key words: DNA methylation, gene transcription, osteoarthritis, genomic regions, promoter varied in subregions of the genome and the effects on transcription were associated with the distribution of DEGs across the genome. The regulation of this appears more complex than initially postulated. Combining the data on epigenetic differences of OA with the genome or transcriptome data for analysis may improve the understanding of the pathophysiological processes of OA. FNDC1 and SCNN1A may potentially be valuable biomarkers for OA.

\section{Introduction}

Osteoarthritis (OA), the most common disease of the joint, is characterized by progressive degeneration of the articular cartilage, resulting in narrowing of joint spaces, osteophytosis and subchondral sclerosis (1). Although OA is a disease with multiple genetic and environmental risk factors, genetics and epigenetics contribute significantly to the pathogenesis and are currently an important subject in the field of OA research (2). Articular chondrocytes are vital for maintaining the homeostasis of the articular cartilaginous tissue and numerous factors may impair their genetic transcriptional function, leading to phenotypic changes and ultimately cartilage degradation (3).

The study of epigenetics involves exploring changes in gene expression in organisms capable of cellular differentiation without changes in the DNA sequence (4). Previous studies have indicated that various types of epigenetics have an impact on the pathogenesis of OA, including DNA methylation, microRNA, long noncoding RNA and histone modification (5). Among these, DNA methylation is an intensively studied epigenetic modifications. The dynamic DNA methylation process is potentiated by DNA methyltransferase (DNMT) enzymes, including DNMT1, 3A and 3B, and the demethylation enzymes ten-eleven translocation methylcytosine dioxygenases (TETs), which include TET1, 2 and 3. DNMTs catalyze the addition of a methyl group to a cytosine-phosphate-guanine dinucleotide $(\mathrm{CpG})$ to form 5-methylcytosine (5mC) (6) .

Studies have demonstrated that DNA methylation may have a key role in regulating gene expression (7), whether at the promoter, enhancer or the gene body (6). In chondrocytes of individuals with OA, the deletion of DNA methylation forms the basis of abnormal expression of certain important catabolic genes, such as the matrix metalloproteinase (MMP), ADAMTS4, IL-1 $\beta$ and NOS2 (8-10). Previous studies have primarily focused on the methylation level at the gene 
promoters, while other regions of DNA methylation, such as gene body and enhancers, warrant further study (11).

In the recent decade, a variety of genome-wide methods for mapping $5 \mathrm{mC}$ have been developed, most commonly by treating the DNA with bisulfite followed by analysis via Illumina $450 \mathrm{~K}$ microarray (12). Through this method, the acquisition of a complete methylated genome has advanced the understanding of the role of DNA methylation in epigenetic processes (11). One of the important advancements in knowledge is that the distribution of methylation across the genome dictates its subsequent expression. Numerous studies have confirmed that methylation of the promoter leads to gene silencing. Specifically, Imagawa et al (13) indicated that a total of $6 \mathrm{CpG}$ sites of the COL9A1 promoter in OA chondrocytes were significantly hypermethylated, while the mRNA expression was significantly decreased. The expression level of COL9A1 mRNA was increased after the treatment with the DNA methylation inhibitor 5-aza-2'-deoxycytidine (5'Aza). On the other hand, the effect of gene body methylation is different from that of promoter methylation, which may be related to transcriptional elongation and selective splicing $(14,15)$. It has been confirmed that gene body methylation is able to activate gene transcription on the X chromosome (16).

In the present study, the data of DNA methylation and mRNA expression of normal and OA cartilage from public datasets were analyzed and compared to identify key genes that may contribute to the pathogenesis of OA. To investigate the functional changes of gene transcription caused by the differences in DNA methylation, the study focused on those genes with differentially methylated sites (DMS) located in the promoter together with their methylation level that was negatively correlated with the mRNA levels, or those genes with DMS localized in the gene body or the 3'UTR together with a positive relationship between methylation levels and gene transcription. Cartilage samples from patients undergoing knee replacement for primary OA were collected to verify the mRNA expression levels of the differentially expressed genes (DEGs), which were then assessed in the in vitro cell experiments to validate the association between DNA methylation and gene transcription.

\section{Materials and methods}

Human articular cartilage samples. Articular cartilage was obtained from 3 knee joint of patients (patient 1: Male, 75 years; patient 2: Female, 69 years; patient 3: Female, 90 years) with OA undergoing knee replacement surgery at Renmin Hospital of Wuhan University (Wuhan, China) in July 2020. Previous studies have confirmed that the outer lateral tibial plateau (oLT), the inner lateral tibial plateau (iLT) and the inner medial tibial plateau (iMT) regions of the knee represent the early, intermediate and late stages of OA (17). Normal and OA cartilage samples were collected from the outer region of the lateral tibial plateau and the inner medial tibial plateau. The present study was approved by the Ethics Committee of Renmin Hospital of Wuhan University (Wuhan, China).

Cell culture. The primary human chondrocytes (cat. no. CP-H096) were purchased from Procell Life Science
\& Technology Co., Ltd. 5'Aza, a DNA methylation inhibitor, was purchased from Aladdin (cat. no. A119533). The primary human chondrocytes were cultured in 90\% DMEM/F12 medium (cat. no. SH30023.01; Hyclone; Cytiva) with added 10\% FBS (Gibco; Thermo Fisher Scientific, Inc.). To gain further insight into the mechanisms of transcriptional regulation by DNA demethylation, the cultured primary chondrocytes were treated with 5'Aza and compared with those that were untreated. The concentration of 5'Aza and cartilage culture time according to those described in previous studies $(18,19)$. Total RNA was then extracted to detect the mRNA expression of the target genes.

Analysis of mRNA expression profiling data. Two mRNA expression profiling datasets, GSE114007 and GSE113825, were obtained from the Gene Expression Omnibus (GEO) database (https://www.ncbi.nlm.nih.gov/gds/). GSE114007 contained 18 normal and 20 OA cartilage samples, while the GSE113825 contained 5 normal and 5 OA cartilage samples from patients without history of OA and patients with OA. The Limma package in R (v3.6.0) was used to identify DEGs between the normal and OA cartilage samples. The cutoff value for DEGs was abs $(\log \mathrm{FC})>1$ and adjusted $\mathrm{P}$-value $<0.05$.

Data normalization and analysis of DNA methylation profiling data. The methylation profiling dataset GSE63695 was obtained from the GEO database (https://www.ncbi.nlm.nih. gov/gds/) and it contained 21 samples of normal cartilage and 23 samples of primary OA cartilage from healthy subjects and patients with OA. Raw intensity data were analyzed by using the ChAMP methylation analysis package in R (v3.6.0) (20). After filtering the unnecessary $\mathrm{CpG}$-including probes with single nucleotide polymorphisms or probes located on $\mathrm{X}$ and $\mathrm{Y}$ chromosomes, the remaining probes were normalized to perform a type-II probe normalization by using Beta Mixture Quantile dilation (21). DMS were defined as CpG sites with absolute (abs) [log fold change (FC) $]>0.1$ and adjusted P-value $<0.05(22,23)$.

Enrichment analysis of $C p G$ sites. The distribution of DMS across the genome may affect their regulation of gene expression. The percentages of DMS and array sites (after filtering) in different subregions and their positions relative to a $\mathrm{CpG}$ island (CGI) were analyzed separately. The seven different subregions included transcription start site (TSS)200, TSS1500, 1st exon, 3'UTR, 5'UTR, intergenic regions and gene body. The positions relative to a CGI included the shore, shelf and opensea regions (24).

Reverse transcription-quantitative PCR (RT-qPCR). RNA was isolated from cartilage tissue using a TRIzol reagent (Invitrogen; Thermo Fisher Scientific, Inc.). Subsequently, RNA was transcribed into complementary (c)DNA using the RevertAid First Strand cDNA Synthesis Kit (cat. no. K1622; Thermo Fisher Scientific, Inc.) according to the manufacturer's protocol. Quantitative analysis of mRNA levels was performed using the FastStart Universal SYBR Green Master Mix (Rox; Roche Diagnostics $\mathrm{GmbH}$ ) according to the manufacturer's protocol. The primer sequences were presented in Table I. GAPDH was used as the internal reference gene. The 
Table I. Primer sequences of the genes used for PCR.

\begin{tabular}{llc}
\hline Gene name & \multicolumn{1}{c}{ Forward $\left(5^{\prime}-3^{\prime}\right)$} & Reverse $\left(5^{\prime}-3^{\prime}\right)$ \\
\hline GAPDH & CAATGACCCCTTCATTGACC & GACAAGCTTCCCGTTCTCAG \\
MAP1B & GGAGCGAGACACTTCGCC & TGATCATTAAGCGCACCTCG \\
FNDC1 & CCACCCAAAGATGCTACCAGT & TTGGGCACTTCCTTTCTGTG \\
ANLN & GGTGTGGTAAGTCCAGAGAGTT & CACCAGATTCAGCTCGAGGG \\
KCNN4 & ATCGGCGCTCTCAATCAAGT & ATTAACAGCCTGCCTCTCGG \\
SCNN1A & CATGAGCAGTATCAAGGGGAA & ACGAGCTTGTCCGAGTTGAG \\
STC2 & CTGTGGAGGTCAGTGGGTGTC & AGCCCAGACAGTACAATGGA
\end{tabular}

Table II. Distribution of DMS and array sites (after filtering) in different subregions.

\begin{tabular}{|c|c|c|c|c|}
\hline CpG subregion & DMS & DMS rate $(\%)$ & $\begin{array}{c}\text { Array sites } \\
\text { (after filtering) }\end{array}$ & $\begin{array}{c}\text { Array rate } \\
\text { (after filtering; \%) }\end{array}$ \\
\hline 3'UTR & 44 & 3 & 13,781 & 3 \\
\hline 5'UTR & 117 & 9 & 35,183 & 9 \\
\hline 1st exon & 20 & 2 & 19,213 & 5 \\
\hline Gene body & 580 & 45 & 130,554 & 34 \\
\hline TSS200 & 41 & 3 & 44,891 & 12 \\
\hline TSS 1500 & 109 & 9 & 55,991 & 14 \\
\hline IGR & 365 & 29 & 87,573 & 23 \\
\hline Island & 121 & 9 & 129,101 & 33 \\
\hline Opensea & 761 & 60 & 131,751 & 34 \\
\hline Shelf & 128 & 10 & 35,245 & 9 \\
\hline Shore & 266 & 21 & 91,089 & 24 \\
\hline
\end{tabular}

DMS, differentially methylated site; CpG, cytosine-phosphate-guanine dinucleotide; IGR, intergenic region; TSS, transcription start site.

thermocycling conditions were as follows: Initial denaturation at $95^{\circ} \mathrm{C}$ for $30 \mathrm{sec}$, followed by 40 cycles of $95^{\circ} \mathrm{C}$ for $15 \mathrm{sec}$ and $60^{\circ} \mathrm{C}$ for $60 \mathrm{sec}$. Relative mRNA expression levels were calculated using the $2^{-\Delta \Delta \mathrm{Cq}}$ method (25).

Statistical analysis. Values are expressed as the mean \pm standard error of the mean. Comparisons between the two groups were performed using the unpaired Student's t-test. GraphPad Prism v.7 (GraphPad Software, Inc.) was used to draw graphs. $\mathrm{P}<0.05$ was considered to indicate statistical significance.

\section{Results}

Screening of DEGs. A total of 1,662 and 1,986 DEGs were identified between the expression profiles of OA and normal human cartilage from the GSE113825 and GSE114007 dataset, respectively. In the GSE113825 dataset, 1,103 DEGs were upregulated and 559 were downregulated in the OA samples, and in the GSE114007 dataset, 1,174 DEGs were upregulated and 812 were downregulated in the OA samples. Of these, further screening revealed that 292 genes shared common differences between the two sets of data, among which 235 were upregulated and 57 were downregulated in OA. The full list of the common DEGs is provided in Table SI. The volcano plots and Venn diagrams of DEGs among GSE113825 and GSE114007 are presented in Fig. 1.

Screening of DMGs. A total of 574 DMS that contained 394 DMGs were identified between the normal and OA cartilage from GSE63695. A complete list of the DMS is provided in Table SII. Among these DMS in the OA cartilage, 220 sites were hypomethylated, while 355 were hypermethylated. Of those 394 DMGs, certain genes that were related to the generation and degradation of extracellular matrix, including ADAMTS17, COL9A1, COL11A2 and COL28A1, and members of the TGF signaling pathway, including BMP6 and SMAD6 (26).

Enrichment analysis of $C p G$ sites. Enrichment analysis was performed for DMS and total array sites (after filtering) across the genome or locations relative to CGIs and the results are provided in Table II. The percentages of DMS and total array sites in the gene body were 45 and $34 \%$, respectively, while the percentages in the 'promoter' regions (including TSS1500, TSS200, 5'UTR and the 1st Exon) were 23 and 40\%, respectively. In addition, $9 \%$ of DMS in comparison with $33 \%$ of total array sites were located on CGIs. These results indicated that the occurrence of differential methylation was related to 

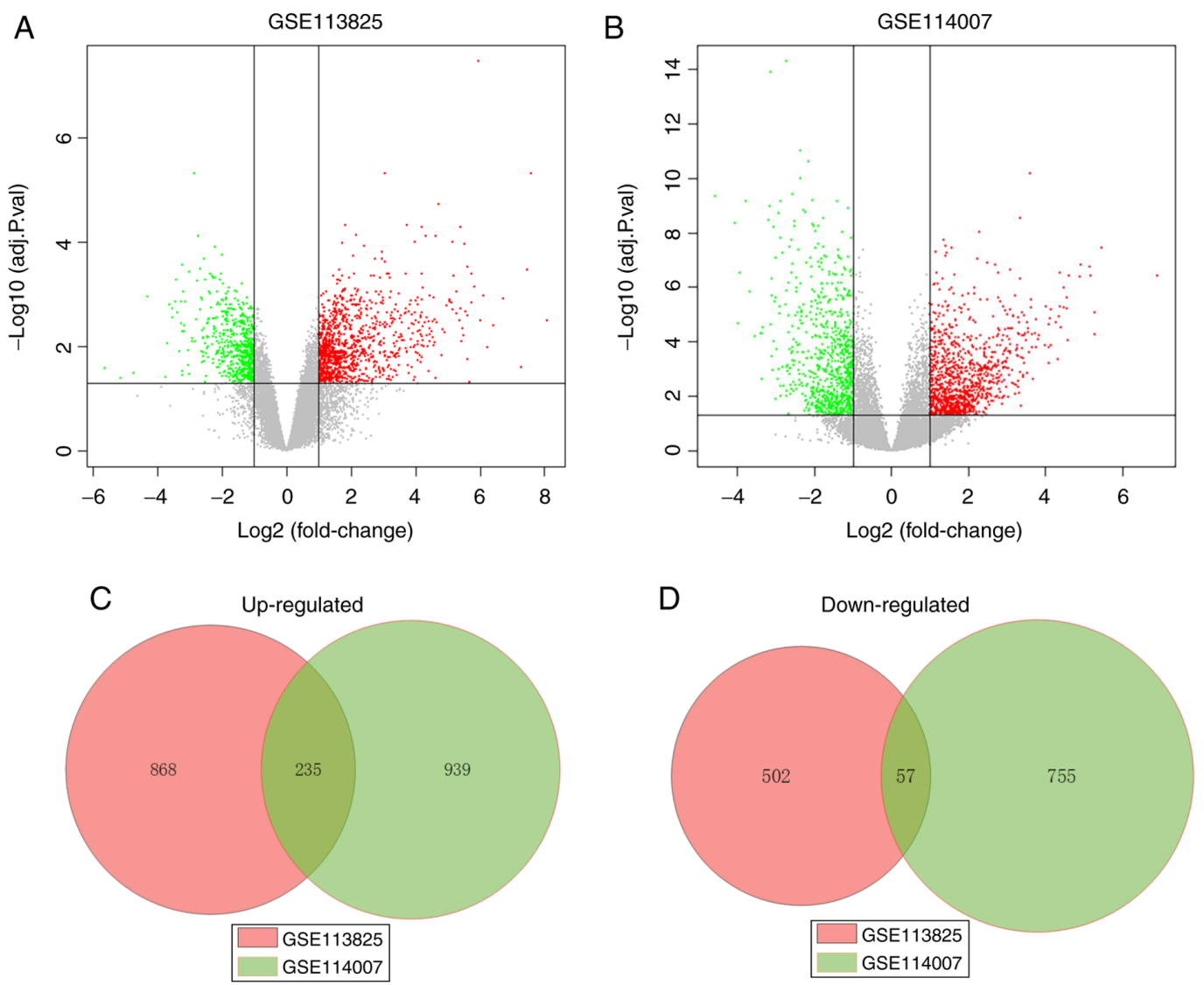

Figure 1. Volcano plots for DEGs between osteoarthritis and normal cartilage from the datasets (A) GSE113825 and (B) GSE114007. Each data-point in the volcano plot represents a gene, with red dots representing upregulated genes and green dots downregulated genes; the grey dots represent genes that were not differentially expressed. (C and D) Venn diagrams of common DEGs among GSE113825 and GSE114007. (C) Upregulated and (D) downregulated DEGs. DEG, differentially expressed gene; P.val, P-value.

the genomic subregions, which appeared more prominent in the regions that had a low $\mathrm{CpG}$ density. A previous study has also demonstrated that CGIs were preferentially observed in the promoter region of genes (27).

Comprehensive analysis of DEGs and DMGs. When comparing DEGs and DMGs, 15 common genes were identified as presented in Table III. Of these, six genes (MAP1B, FNDC1, ANLN, KCNN4, SCNN1A and STC2) were further investigated. The DMS of STC2 and SCNN1A were located in the promoter, with the level of methylation negatively associated with gene transcription. On the other hand, the DMS of MAP1B, FNDC1, ANLN and KCNN4 were located in the gene body, with the level of methylation positively associated with gene transcription.

Validation of screened genes. The mRNA expression of those selected screened genes was verified by RT-qPCR, which indicated that in the OA cartilage, MAP1B, FNDC1 and ANLN were upregulated, while SCNN1A and STC2 were downregulated $(\mathrm{P}<0.05, \mathrm{P}<0.01$ or $\mathrm{P}<0.001$; Fig. 2). No significant alteration in the mRNA levels of ANLN was observed. This was consistent with the results obtained from the mRNA expression datasets.

Changes in gene expression in cultured chondrocytes after DNA demethylation. To investigate whether hypomethylation

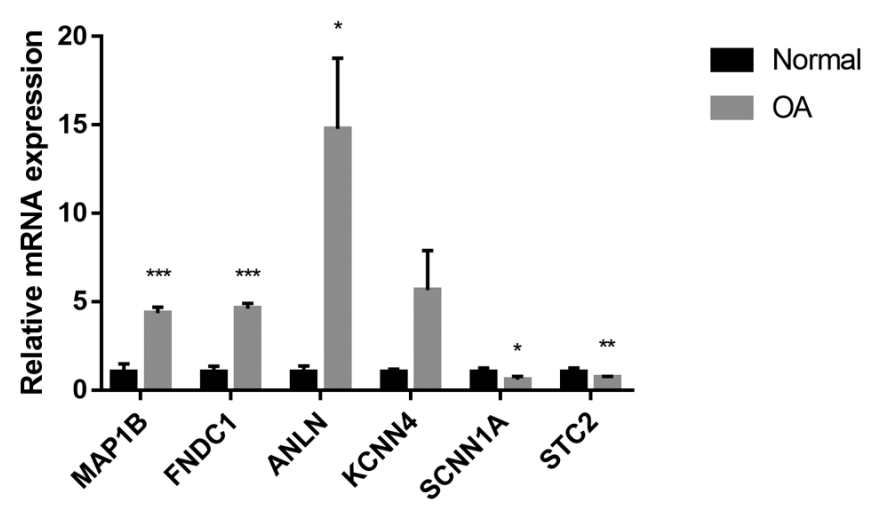

Figure 2. Relative mRNA expression of screened genes in normal and OA knee articular cartilage. Values are expressed as the mean \pm standard error of the mean. ${ }^{*} \mathrm{P}<0.05,{ }^{* *} \mathrm{P}<0.01,{ }^{* * *} \mathrm{P}<0.001$ vs. normal group. OA, osteoarthritis.

affected the transcription of those genes, 5'Aza, a DNA methylation inhibitor (28), was applied as a useful tool in a further experiment. The effects of 5'Aza on the expression of the MAP1B, FNDC1, ANLN, KCNN4, SCNN1A and STC2 genes in cultured human articular chondrocytes were assessed. As indicated in Fig. 3, the expression of FNDC1 and SCNN1A was downregulated after 5'Aza treatment $(\mathrm{P}<0.05$ and $\mathrm{P}<0.001$, respectively). No significant alterations in the mRNA levels of MAP1B, ANLN, KCNN4 and STC2 were observed. 
Table III. Differentially expressed genes harboring differentially methylated sites between OA and normal knee articular cartilage.

A, Hypermethylated in $\mathrm{OA}$

\begin{tabular}{lll}
\hline Gene symbol & $\begin{array}{c}\text { Gene expression } \\
\text { status in OA }\end{array}$ & $\begin{array}{c}\text { CpG genomic } \\
\text { feature }\end{array}$ \\
\hline ANPEP & Upregulated & 5'UTR \\
MAP1B & Upregulated & Body \\
FNDC1 & Upregulated & Body \\
TPPP3 & Upregulated & TSS1500 \\
ANLN & Upregulated & Body \\
BMPR1B & Upregulated & 5'UTR \\
KCNN4 & Upregulated & Body \\
SCNN1A & Downregulated & 5'UTR \\
STC2 & Downregulated & TSS1500
\end{tabular}

$\mathrm{B}$, Hypomethylated in $\mathrm{OA}$

\begin{tabular}{lll}
\hline Gene symbol & $\begin{array}{c}\text { Gene expression } \\
\text { status in OA }\end{array}$ & $\begin{array}{c}\text { CpG genomic } \\
\text { feature }\end{array}$ \\
\hline TRPV2 & Upregulated & Body \\
LHFPL2 & Upregulated & Body \\
IGFBP4 & Upregulated & Body \\
BTBD11 & Upregulated & Body \\
CAPZB & Upregulated & Body \\
AQP1 & Upregulated & 1st exon, body \\
\hline
\end{tabular}

OA, osteoarthritis; $\mathrm{CpG}$, cytosine-phosphate-guanine dinucleotide.

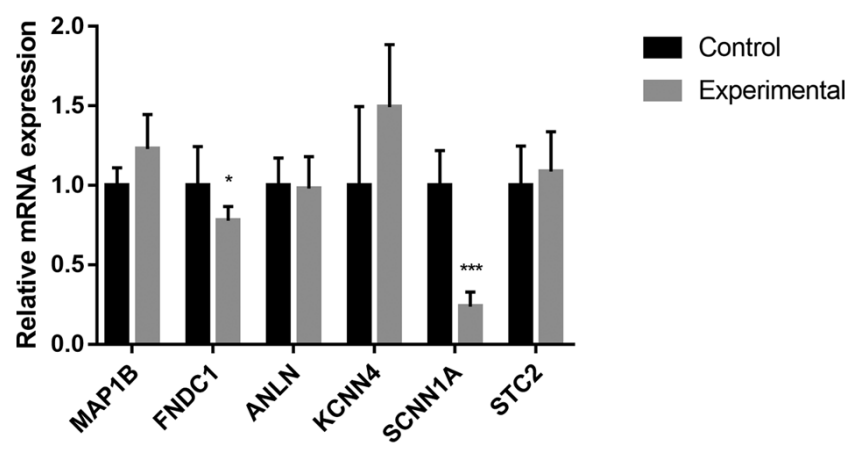

Figure 3. Relative mRNA expression of screened genes in cultured primary human chondrocytes $(\mathrm{n}=3)$ treated with 5 -aza-2'-deoxycytidine $(10 \mu \mathrm{M})$ or vehicle for 4 days. Values are expressed as the mean \pm standard error of the mean. ${ }^{*} \mathrm{P}<0.05,{ }^{* * * *} \mathrm{P}<0.001$ vs. control.

\section{Discussion}

In the present study, both the DNA methylation data and mRNA expression data of OA and normal cartilage were analyzed to identify genes that may have a role in the pathogenesis of the disease. A total of two mRNA expression datasets were used to accurately identify DEGs. The methylation profiling dataset GSE63695 obtained from GEO database used for the Illumina Human Methylation 450 BeadChip microarray. The array typically measures a total of $485,512 \mathrm{CpG}$ sites and its probes have a wide range of genomic characteristics, including enhancers, promoters, UTR and gene body. This may provide a broader understanding of the epigenetic changes that occur in the pathogenesis of OA when compared with the Illumina 27K methylation microarray (29).

Previous studies have indicated that differential mRNA expression of certain key genes in the pathogenesis of OA is associated with changes in their epigenetic status, such as COL9A1. COL9A1 encodes type IX collagen, which is paramount in the formation of a collagen network that maintains the integrity of cartilage tissue while protecting cartilage tissue from mechanical damage $(30,31)$. The study by Imagawa et al (13) indicated that hypermethylation of the COL9A1 promoter resulted in its downregulation in OA cartilage tissue. Indeed, studies have revealed numerous distinct DMS between OA and normal tissue, but only a small fraction of these sites have been indicated to affect gene expression. To date, in OA, the impact of DMS on changes to articular cartilage-related gene expression and the specific mechanisms have remained to be fully elucidated.

DMS between normal and OA cartilage may be distinguished by their genomic features such as promoters, enhancers, UTR and gene bodies. In the present study, an enrichment analysis was performed for DMS and total array sites (after filtering) across the genome. Most of the DMS were enriched in the intergenic region and gene body, while only $23 \%$ were enriched in the promoter (32). Furthermore, when the positions of the DMS relative to a CGI were analyzed, the percentage of DMS on CGIs was small, indicating that regions lacking CGIs, such as the gene body and enhancer subregions, were more likely to undergo changes in methylation levels than promoters that were rich in CGIs.

Methylation of the promoter primarily imposes a negative regulatory effect on transcription when compared with methylation of the gene body $(24,33)$. The 1st exon has been considered as an important negative factor in the regulation of transcription (34). In the present study, 15 common key genes were identified and six of these were verified, namely MAP1B, FNDC1, ANLN, KCNN4, SCNN1A and STC2. First, mRNAs were extracted from the knee cartilage tissues of patients with OA. RT-qPCR was then performed to validate the expression levels of mRNA of these six genes in OA cartilage. Subsequently, validation of these genes was performed in vitro using primary chondrocytes cultured with 5'Aza or vehicle, revealing that only STC2 and FNDC1 had met our expectations; DMS located in the promoter was negatively associated with mRNA levels, while DMS located in the gene body or the 3'UTR was positively associated with gene transcription.

The present study suggested a relationship between methylation levels and gene expression, which appears more complex than initially postulated. Although methylation of promoter regions has been associated with silencing of the downstream gene expression, it has long been debated whether it is silencing or methylation that comes first (11). Of note, the study by Keller et al (35) has indicated that the role of DNA methylation at the promoter regions of $C$. intestinalis was related to the methylation states of the nearby 
gene body regions. Furthermore, promoter methylation adjacent to the methylated gene body was negatively correlated with gene expression levels, but this phenomenon was not observed when not in the direct vicinity to the methylated gene body (35).

The role of gene body methylation has remained to be fully elucidated $(36,37)$. It has long been postulated that gene body DNA methylation suppresses spurious transcription in the coding region, given that DNA methylation is usually repressive (38). Through this, gene body methylation may effectively inhibit 'transcriptional noise', meaning that gene expression levels between cells may vary despite the same genetic materials and biological conditions (36). This hypothesis, if true, would account for the consistent positive correlation between the methylation of genes and their transcriptional levels. In addition, other studies have suggested that gene body DNA methylation may function in conjunction with other epigenetic modifications. For instance, the initiation and elongation of forged transcripts are inhibited by excluding the occupation of RNA polymerase II in addition to recruiting multiple inhibitory histone markers (39). The study by Jjingo et al (37) also has uncovered the relationship between gene body methylation and expression levels, which appeared bell-shaped rather than monotonic. Hence, a model has been proposed that may explain the relationship as a result of interactions between chromatin openness and RNA polymerase II density, in which genes with low or high expression had the lowest methylation level, while genes with medium expression had the highest methylation level (37).

The present results may also be explained by other studies. For instance, Den Hollander et al (40) postulated a novel theory that epigenetic regulation only affects the transcription of certain genes, such as genes involved in maintaining the phenotype of chondrocytes. In addition, Imagawa et al (13) suggested that the mRNA levels of COL2A1 in OA chondrocytes were $>10$-fold higher than those in the control group, but this was not mediated by changes in the methylation status of gene promoters or enhancers. However, it may be inferred that the fold changes of mRNA expression does not necessarily contribute to higher significance. Furthermore, setting a threshold of fold change to screen for differential genes may exclude certain genes with small differential multiples but have an important functional role.

The present results concerning FNDC1, which may represent a bone metabolism-related factor (41), were novel. It was previously reported that inhibition of FNDC1 expression in bone marrow mesenchymal stem cells resulted in the impediment of postmenopausal osteoporosis (41). However, to date, there has been no clear evidence to suggest the role of FNDC1 in cartilage tissue in the pathogenesis of OA. Based on several previous studies, FNDC1 may share similar functions to fibronectin (FN), which is a high-molecular-weight glycoprotein comprised of three types of repeating amino acid units, type I, II and III repeats (42). The function of FN varies with the tissue type and fibronectin serves as a scaffold for cell adhesion and migration that contributes to the healing of skin wounds (43). However, in the cartilage tissues of OA, if the removal of fibronectin is not complete, the persistence of fibronectin fragments or aggregates impairs tissue regeneration and remodeling. When degraded into multiple fragments by proteases, FN may bind to Clq of the complement system, which may lead to chronic leukocyte stimulation $(44,45)$. In addition, FN fragments may inhibit the synthesis of cartilage matrix such as sulfated proteoglycans and stimulate the release of proinflammatory cytokines and MMP, thus mediating cartilage injury in OA (42). Thus, FNDC1 may potentially be a valuable biomarker for OA.

In the present study, certain key genes associated with the distribution of DMS across the genome were identified and further validated via in vitro experiments. The present results suggested that the regulation of gene transcription by DNA methylation may not have a relatively fixed rule. However, compared with the conventional genome-wide DNA methylation analysis, this may improve the current understanding of the function of DNA methylation and its regulation of transcription. Nevertheless, the establishment of a causal relationship remains a great challenge in epigenetics research (23). Further mechanistic studies, such as large-scale longitudinal interference in animal experiments, are therefore warranted. In addition, for the key genes identified in the present study, further analysis and functional verification will be performed in the future. For instance, alterations in the binding status of transcription factors to genes following changes in DNA methylation levels may be detected by a chromatin immunoprecipitation assay. Alternatively, by knocking out or overexpressing these genes, the effect of these changes on chondrocyte or tissue phenotype and the specific regulatory mechanisms may be observed.

There were certain limitations to the present study. First, the methylation level of target genes prior to and after 5'Aza treatment was not investigated. Undoubtedly, the assessment of changes in methylation levels may assist in determining the role of 5'Aza in affecting the methylation levels more accurately and may provide a clearer presumption that it was the changes in DNA methylation levels that regulated the transcription of genes. However, according to previous studies, this part is non-essential $(18,46)$. Furthermore, most previous studies have indicated that 5 'Aza accurately demethylates the DNA of cells $(28,47)$. Therefore, the measurement of the methylation level of target genes concerning 5'Aza treatment is considered non-essential. As another limitation, the analysis of two mRNA expression data using the intersection method to obtain common differential genes lacks novelty (22). Finally, the sample size of patients from whom articular cartilage tissue was obtained and the sample size of the DNA methylation and mRNA expression data obtained from the GEO database were small.

In the in vitro experiments involving culture of human chondrocytes, glucosamine has been indicated to prevent cytokine-induced demethylation of the promoter of IL-1 $\beta$, resulting in decreased IL-1 $\beta$ expression. This suggests that modification of DNA methylation may be a potential therapeutic strategy to intervene in the OA process (9).

In conclusion, in the present study, a group of key genes in OA regulated by DNA methylation was identified and several of them were validated. The present results provide an enhanced understanding of the regulation of gene transcription of these key genes by epigenetics in OA, which may serve as potential biomarkers in the pathogenesis of $\mathrm{OA}$ and will 
provide avenues for targeted therapeutic intervention of OA pathogenesis through modification of DNA methylation.

\section{Acknowledgements}

Not applicable.

\section{Funding}

The authors are grateful for the financial support from the National Natural Science Foundation of China (grant no. 81071494) and Hubei Provincial Science and Technology Support Program (grant no. 2015BCA316).

\section{Availability of data and materials}

The datasets used and/or analyzed during the current study are available from the corresponding author on reasonable request.

\section{Authors' contributions}

PY and BQ designed the study. PY, XX and JY acquired and interpreted the data. PY and XX analyzed the data and were major contributors in writing the manuscript. BQ prepared the manuscript and supervised the study. BQ and XX approve the authenticity of all the raw data. All authors read and approved the final manuscript.

\section{Ethics approval and consent to participate}

The present study was approved by the Ethics Committee of the Renmin Hospital of Wuhan University (Wuhan, China).

\section{Patient consent for publication}

Not applicable.

\section{Competing interests}

The authors declare that they have no competing interests.

\section{References}

1. Miranda-Duarte A: DNA methylation in osteoarthritis: Current status and therapeutic implications. Open Rheumatol J 12: 37-49, 2018.

2. Jeffries MA: Osteoarthritis year in review 2018: Genetics and epigenetics. Osteoarthritis Cartilage 27: 371-377, 2019.

3. Lotz M and Loeser RF: Effects of aging on articular cartilage homeostasis. Bone 51: 241-248, 2012.

4. Goldberg AD, Allis CD and Bernstein E: Epigenetics: A landscape takes shape. Cell 128: 635-638, 2007.

5. Barter MJ, Bui C and Young DA: Epigenetic mechanisms in cartilage and osteoarthritis: DNA methylation, histone modifications and microRNAs. Osteoarthritis Cartilage 20: 339-349, 2012.

6. Shen J, Abu-Amer Y, O'Keefe RJ and McAlinden A: Inflammation and epigenetic regulation in osteoarthritis. Connect Tissue Res 58: 49-63, 2017.

7. Im GI and Choi YJ: Epigenetics in osteoarthritis and its implication for future therapeutics. Expert Opin Biol Ther 13: 713-721, 2013.

8. Roach HI, Yamada N, Cheung KS, Tilley S, Clarke NM, Oreffo RO, Kokubun $\mathrm{S}$ and Bronner F: Association between the abnormal expression of matrix-degrading enzymes by human osteoarthritic chondrocytes and demethylation of specific $\mathrm{CpG}$ sites in the promoter regions. Arthritis Rheum 52: 3110-3124, 2005.
9. Imagawa $\mathrm{K}$, de Andres MC, Hashimoto K, Pitt D, Itoi E, Goldring MB, Roach HI and Oreffo RO: The epigenetic effect of glucosamine and a nuclear factor-kappa B $(\mathrm{NF}-\kappa \mathrm{B})$ inhibitor on primary human chondrocytes-implications for osteoarthritis. Biochem Biophys Res Commun 405: 362-367, 2011.

10. Hashimoto K, Oreffo RO, Gibson MB, Goldring MB and Roach HI: DNA demethylation at specific $\mathrm{CpG}$ sites in the IL1 $\beta$ promoter in response to inflammatory cytokines in human articular chondrocytes. Arthritis Rheum 60: 3303-3313, 2009.

11. Jones PA: Functions of DNA methylation: Islands, start sites, gene bodies and beyond. Nat Rev Genet 13: 484-492, 2012.

12. Harris RA, Wang T, Coarfa C, Nagarajan RP, Hong C, Downey SL, Johnson BE, Fouse SD, Delaney A, Zhao Y, et al: Comparison of sequencing-based methods to profile DNA methylation and identification of monoallelic epigenetic modifications. Nat Biotechnol 28: 1097-1105, 2010.

13. Imagawa $\mathrm{K}$, de Andres MC, Hashimoto K, Itoi E, Otero M, Roach HI, Goldring MB and Oreffo RO: Association of reduced type IX collagen gene expression in human osteoarthritic chondrocytes with epigenetic silencing by DNA hypermethylation. Arthritis Rheumatol 66: 3040-3051, 2014.

14. Hahn MA, Wu X, Li AX, Hahn T and Pfeifer GP: Relationship between gene body DNA methylation and intragenic H3K9me 3 and H3K36me 3 chromatin marks. PLoS One 6: e18844, 2011.

15. Laurent L, Wong E, Li G, Huynh T, Tsirigos A, Ong CT, Low HM, Kin Sung KW, Rigoutsos I, Loring J and Wei CL: Dynamic changes in the human methylome during differentiation. Genome Res 20: 320-331, 2010.

16. Hellman A and Chess A: Gene body-specific methylation on the active X chromosome. Science 315: 1141-1143, 2007.

17. Chou CH, Lee CH, Lu LS, Song IW, Chuang HP, Kuo SY, Wu JY, Chen YT, Kraus VB, Wu CC and Lee MT: Direct assessment of articular cartilage and underlying subchondral bone reveals a progressive gene expression change in human osteoarthritic knees. Osteoarthritis Cartilage 21: 450-461, 2013.

18. Zhao L, Wang Q, Zhang C and Huang C: Genome-wide DNA methylation analysis of articular chondrocytes identifies TRAF1, CTGF, and CX3CL1 genes as hypomethylated in osteoarthritis. Clin Rheumatol 36: 2335-2342, 2017.

19. Iliopoulos D, Malizos KN and Tsezou A: Epigenetic regulation of leptin affects MMP-13 expression in osteoarthritic chondrocytes: Possible molecular target for osteoarthritis therapeutic intervention. Ann Rheum Dis 66: 1616-1621, 2007.

20. Morris TJ, Butcher LM, Feber A, Teschendorff AE, Chakravarthy AR, Wojdacz TK and Beck S: ChAMP: 450k chip analysis methylation pipeline. Bioinformatics 30: 428-430, 2014.

21. Teschendorff AE, Marabita F, Lechner M, Bartlett T, Tegner J, Gomez-Cabrero D and Beck S: A beta-mixture quantile normalization method for correcting probe design bias in Illumina Infinium 450 k DNA methylation data. Bioinformatics 29: 189-196, 2013.

22. Li Z, Zhang R, Yang X, Zhang D, Li B, Zhang D, Li Q and Xiong Y: Analysis of gene expression and methylation datasets identified ADAMTS9, FKBP5, and PFKBF3 as biomarkers for osteoarthritis. J Cell Physiol 234: 8908-8917, 2019.

23. Rushton MD, Reynard LN, Barter MJ, Refaie R, Rankin KS, Young DA and Loughlin J: Characterization of the cartilage DNA methylome in knee and hip osteoarthritis. Arthritis Rheumatol 66: 2450-2460, 2014.

24. Mishra NK and Guda C: Genome-wide DNA methylation analysis reveals molecular subtypes of pancreatic cancer. Oncotarget 8: 28990-29012, 2017.

25. Livak KJ and Schmittgen TD: Analysis of relative gene expression data using real-time quantitative PCR and the 2(-Delta Delta C(T)) method. Methods 25: 402-408, 2001.

26. Jung SM, Lee JH, Park J, Oh YS, Lee SK, Park JS, Lee YS, Kim JH, Lee JY, Bae YS, et al: Smad6 inhibits non-canonical TGF- $\beta 1$ signalling by recruiting the deubiquitinase A20 to TRAF6. Nat Commun 4: 2562, 2013.

27. Brena RM, Huang TH and Plass C: Toward a human epigenome. Nat Genet 38: 1359-1360, 2006.

28. Kim KI, Park YS and Im GI: Changes in the epigenetic status of the SOX-9 promoter in human osteoarthritic cartilage. J Bone Miner Res 28: 1050-1060, 2013.

29. Wright ML, Dozmorov MG, Wolen AR, Jackson-Cook C, Stark weather AR, Lyon DE and York TP: Establishing an analytic pipeline for genome-wide DNA methylation. Clin Epigenetics 8: 45, 2016. 
30. Mustafa Z, Chapman K, Irven C, Carr AJ, Clipsham K, Chitnavis J, Sinsheimer JS, Bloomfield VA, McCartney M, Cox $\mathrm{O}$, et al: Linkage analysis of candidate genes as susceptibility loci for osteoarthritis-suggestive linkage of COL9A1 to female hip osteoarthritis. Rheumatology (Oxford) 39: 299-306, 2000.

31. Loughlin J, Mustafa Z, Dowling B, Southam L, Marcelline L, Raina SS, Ala-Kokko L and Chapman K: Finer linkage mapping of a primary hip osteoarthritis susceptibility locus on chromosome 6. Eur J Hum Genet 10: 562-568, 2002.

32. Li K, Qin L, Jiang S, Li A, Zhang C, Liu G, Sun J, Sun H, Zhao Y, Li N and Zhang Y: The signature of HBV-related liver disease in peripheral blood mononuclear cell DNA methylation. Clin Epigenetics 12: 81, 2020.

33. Gutierrez-Arcelus M, Lappalainen T, Montgomery SB, Buil A, Ongen H, Yurovsky A, Bryois J, Giger T, Romano L, Planchon A, et al: Passive and active DNA methylation and the interplay with genetic variation in gene regulation. Elife 2 : e00523, 2013.

34. Brenet F, Moh M, Funk P, Feierstein E, Viale AJ, Socci ND and Scandura JM: DNA methylation of the first exon is tightly linked to transcriptional silencing. PLoS One 6: e14524, 2011.

35. Keller TE, Han P and Yi SV: Evolutionary transition of promoter and gene body DNA methylation across invertebrate-vertebrate boundary. Mol Biol Evol 33: 1019-1028, 2016.

36. Huh I, Zeng J, Park T and Yi SV: DNA methylation and transcriptional noise. Epigenetics Chromatin 6: 9, 2013.

37. Jjingo D, Conley AB, Yi SV, Lunyak VV and Jordan IK: On the presence and role of human gene-body DNA methylation. Oncotarget 3: 462-474, 2012

38. Bird AP and Wolffe AP: Methylation-induced repression-belts, braces, and chromatin. Cell 99: 451-454, 1999.

39. Lorincz MC, Dickerson DR, Schmitt M and Groudine $M$ Intragenic DNA methylation alters chromatin structure and elongation efficiency in mammalian cells. Nat Struct Mol Biol 11: 1068-1075, 2004.
40. Den Hollander W, Ramos YF, Bomer N, Elzinga S, Van der Breggen R, Lakenberg N, De Dijcker WJ, Suchiman HE, Duijnisveld BJ, Houwing-Duistermaat JJ, et al: Transcriptional associations of osteoarthritis-mediated loss of epigenetic control in articular cartilage. Arthritis Rheumatol 67: 2108-2116, 2015.

41. Xiao Y, Wei R, Yuan Z, Lan X, Kuang J, Hu D, Song Y and Luo J: Rutin suppresses FNDC1 expression in bone marrow mesenchymal stem cells to inhibit postmenopausal osteoporosis. Am J Transl Res 11: 6680-6690, 2019.

42. Stoffels JM, Zhao C and Baron W: Fibronectin in tissue regeneration: Timely disassembly of the scaffold is necessary to complete the build. Cell Mol Life Sci 70: 4243-4253, 2013.

43. Pankov R and Yamada KM: Fibronectin at a glance. J Cell Sci 115: 3861-3863, 2002.

44. Bing DH, Almeda S, Isliker H, Lahav J and Hynes RO: Fibronectin binds to the Clq component of complement. Proc Natl Acad Sci USA 79: 4198-4201, 1982.

45. Carsons SE, Schwartzman S, Diamond HS and Berkowitz E: Interaction between fibronectin and $\mathrm{Clq}$ in rheumatoid synovial fluid and normal plasma. Clin Exp Immunol 72: 37-42, 1988.

46. Alvarez-Garcia O, Fisch KM, Wineinger NE, Akagi R, Saito M, Sasho T, Su AI and Lotz MK: Increased DNA methylation and reduced expression of transcription factors in human osteoarthritis cartilage. Arthritis Rheumatol 68: 1876-1886, 2016

47. Christman JK: 5-Azacytidine and 5-aza-2'-deoxycytidine as inhibitors of DNA methylation: Mechanistic studies and their implications for cancer therapy. Oncogene 21: 5483-5495, 2002. International (CC BY-NC-ND 4.0) License. 